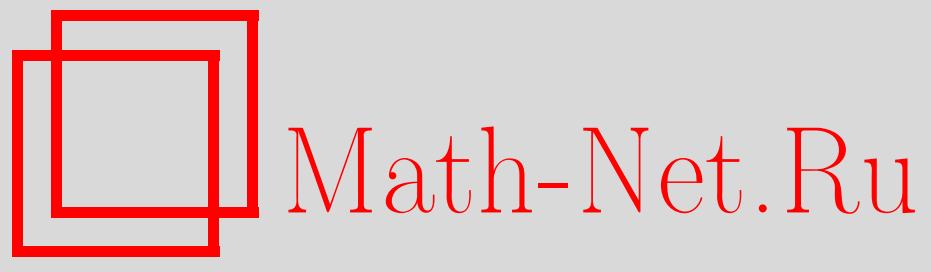

О. И. Кузнецова, Об одном кратном тригонометрическом ряде, Матем. заметки, 2010, том 88, выпуск 6, 950-952

DOI: https://doi.org/10.4213/mzm8297

Использование Общероссийского математического портала Math-Net.Ru подразумевает, что вы прочитали и согласны с пользовательским соглашением http://www . mathnet.ru/rus/agreement

Параметры загрузки:

IP : 34.229 .108 .108

26 апреля 2023 г., 13:15:31

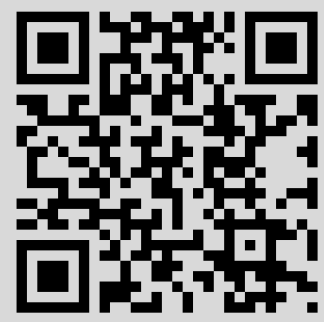




\section{Об одном кратном тригонометрическом ряде}

\section{О. И. Кузнецова}

Пусть $\mathbb{R}^{m}-m$-мерное вещественное евклидово пространство, $x=\left(x_{1}, \ldots, x_{m}\right) \in \mathbb{R}^{m}$, $x y=x_{1} y_{1}+\cdots+x_{m} y_{m}-$ скалярное произведение векторов $x$ и $y$,

$$
\mathbb{T}^{m}=\left\{x \in \mathbb{R}^{m}:-\pi \leqslant x_{i}<\pi, i=1, \ldots, m\right\}
$$

- $m$-мерный тор, $\mathbb{Z}_{+}^{m}$ - множество векторов $l$ из $\mathbb{R}^{m}$ с целыми неотрицательными координатами, $|l|_{1}=l_{1}+\cdots+l_{m}$.

В [1] рассматривается ряд

$$
\sum_{k=0}^{\infty} a_{k} F_{k}(x)=\sum_{k=0}^{\infty} a_{k} \sum_{|l|_{1}=k} e^{i l x}, \quad x \in \mathbb{T}^{m} .
$$

Предполагается, что последовательность коэффициентов $a=\left\{a_{k}\right\}_{k=0}^{\infty}$ ряда (1) удовлетворяет условиям Боаса-Теляковского [2]:

$$
\begin{gathered}
a_{k} \rightarrow 0, \quad k \rightarrow \infty, \\
T(a)=\sum_{k=0}^{\infty}\left|\Delta a_{k}\right|+\sum_{k=2}^{\infty}\left|\sum_{l=1}^{[k / 2]} \frac{1}{l}\left(\Delta a_{k-1}-\Delta a_{k+1}\right)\right|<\infty,
\end{gathered}
$$

где $\Delta a_{k}=a_{k}-a_{k+1}$. Утверждается [1; теорема], что при выполнении условий (2), (3) ряд (1) сходится почти всюду к некоторой функции $f \in L\left(\mathbb{T}^{m}\right)$ и является ее рядом Фурье тогда и только тогда, когда

$$
\sum_{k=1}^{\infty} \frac{\left|a_{k}\right|}{k}<\infty .
$$

Данная теорема при $m=2,3, \ldots$ не верна. Покажем это.

Если функция $f \in L\left(\mathbb{T}^{m}\right)$ имеет ряд Фурье степенного типа $\left(\widehat{f}(l)=0\right.$ при $\left.l \notin \mathbb{Z}_{+}^{m}\right)$, то для нее справедлив $m$-мерный аналог неравенства Харди-Литтлвуда [3; раздел 4.3]. Для суммы ряда (1) это неравенство имело бы вид $\left(\|\cdot\|_{L\left(\mathbb{T}^{m}\right)}=\|\cdot\|\right)$

$$
\|f\| \geqslant \frac{1}{\pi} \sum_{k=0}^{\infty} \frac{\left|a_{k}\right|}{k+1}\left\|F_{k}\right\| .
$$

Множество точек

$$
\left\{x \in \mathbb{R}^{m}: x_{i} \geqslant 0, i=1, \ldots, m, \sum_{i=1}^{m} x_{i}=k\right\}
$$

выпукло в $\mathbb{R}^{m-1}$ и содержит $(m-1)$-мерный шар радиуса $c k$, где $c=c(m)$ - некоторая положительная константа. По теореме Юдина [4]

$$
\left\|F_{k}\right\| \geqslant c_{1}(m) \log ^{m-1}(k+2) .
$$

Получаем оценку снизу $\|f\|$ :

$$
\|f\| \geqslant c_{2}(m) \sum_{k=0}^{\infty} \frac{\left|a_{k}\right|}{k+1} \log ^{m-1}(k+2) .
$$

(С) О.И. КУЗНЕцовА, 2010 
Пусть

$$
a_{k}=\frac{1}{\log ^{1+\varepsilon}(k+2)}, \quad k=0,1, \ldots, \quad 0<\varepsilon<1 .
$$

Данная выпуклая последовательность удовлетворяет условиям (2)-(4), но ряд (5) для нее расходится при $m \geqslant 2$.

Заметим, что ошибочным в доказательстве теоремы является утверждение, что

$$
\int_{\mathbb{T}^{m}}\left|\sum_{k=0}^{2 n} \lambda_{k}^{(n)} F_{k}(x)\right| d x \leqslant c<\infty
$$

где

$$
\lambda_{k}^{(n)}= \begin{cases}1, & k=0, \ldots, n \\ \frac{2 n-k+1}{n+1}, & k=n+1, \ldots, 2 n .\end{cases}
$$

Из неравенства (5) при $a_{k}=\lambda_{k}^{(n)}$ следует, что данный интеграл не меньше, чем

$$
M \sum_{k=0}^{n} \frac{\log ^{m-1}(k+2)}{k+1} \geqslant M_{1} \log ^{m} n
$$

где $M, M_{1}>0$.

Приведем простое достаточное условие интегрируемости ряда (1). Пусть

$$
S_{n}(x)=\sum_{k=0}^{n} a_{k} F_{k}(x)
$$

- частичная сумма ряда (1).

УТВЕРЖДЕНИЕ. Если коэффициенты ряда (1) удовлетворяют условию (2) u

$$
\sum_{k=0}^{\infty}\left|\Delta a_{k}\right| \log ^{m}(k+2)<\infty
$$

то ряд (1) сходится почти всюду к некоторой функиии $f \in L\left(\mathbb{T}^{m}\right)$, является ее рядом Фуръе, а соотношение

$$
\lim _{n \rightarrow \infty} \int_{\mathbb{T}^{m}}\left|f(x)-S_{n}(x)\right| d x=0
$$

выполняется тогда и только тогда, когда

$$
a_{n}=o\left(\frac{1}{\log ^{m} n}\right), \quad n \rightarrow \infty .
$$

ДокАзАтельство. Обозначим $D_{n}(x)=\sum_{|l|_{1} \leqslant n} e^{i l x}$. Применим к частичной сумме $S_{n}$ преобразование Абеля

$$
S_{n}(x)=\sum_{k=0}^{n-1} \Delta a_{k} D_{k}(x)+a_{n} D_{n}(x) .
$$

Из условия (6) следует, что

$$
\sum_{k=0}^{\infty}\left|\Delta a_{k}\right|<\infty
$$

Поскольку $a_{n} \rightarrow 0$ при $n \rightarrow \infty$, а последовательность $D_{n}$ ограничена почти всюду на $\mathbb{T}^{m}$ (см., например, [5; лемма 2]), в равенстве (9) при почти всех $x$ можно перейти к пределу при $n \rightarrow \infty$. Пусть

$$
f(x) \stackrel{\text { def }}{=} \sum_{k=0}^{\infty} a_{k} F_{k}(x)=\sum_{k=0}^{\infty} \Delta a_{k} D_{k}(x)
$$


Интегрируемость функции $f$ следует из верхней оценки в двустороннем неравенстве

$$
\int_{\mathbb{T}^{m}}\left|D_{n}(x)\right| d x \asymp \log ^{m} n
$$

([6], см. также [7], - оценка сверху, [4] - нижняя оценка) и условия (6). Доказательство того факта, что ряд (1) есть ряд Фурье, стандартно (см., например, [5]). Необходимость и достаточность условия (8) для сходимости данного ряда в $L\left(\mathbb{T}^{m}\right)$ (соотношение (7)) вытекает из равенств (9) и (10), условия (6) и двусторонней оценки (11).

В заключение отметим, что если $\left\{a_{k}\right\}$ - монотонная последовательность, то достаточное условие (6) и необходимое условие (5) интегрируемости ряда (1) совпадают.

\section{СПИСОК ЦИТИРОВАННОЙ ЛИТЕРАТУРЫ}

[1] П. В. Задерей, Е. Н. Пелагенко, Матем. заметки, 84:4 (2008), 627-631. [2] С. А. Теляковский, Изв. АН СССР. Сер. матем., 28:6 (1964), 1209-1236. [3] У. Рудин, Теория функиий в поликруге, Мир, М., 1974. [4] В.А. Юдин, Матем. заметки, 25:1 (1979), 119-122. [5] О.И. Кузнецова, Матем. заметки, 63:3 (1998), 402-406. [6] Э. С. Белинский, "Поведение констант Лебега некоторых методов суммирования кратных рядов Фурье", Метрические вопросы теории функиий и отображений, Наукова думка, Киев, 1977, 19-39. [7] А.Н. Подкорытов, Вестн. ЛГУ. Матема., мех., астрон., 1982, № 7, $110-111$.

\section{О. И. Кузнецова}

Поступило

Институт прикладной математики

и механики НАН Украины,

г. Донецк

E-mail: kuznets@iamm.ac.donetsk.ua 